\title{
Evaluating the Components of Alexithymia in Patients With Obsessive-Compulsive Disorder
}

\author{
Mina Bozorg ${ }^{*}$, Changiz Rahimi $^{\circledR}$, Nurallah Mohammadi ${ }^{\circledR}$ \\ Department of Clinical Psychology, Shiraz University, Shiraz, Iran
}

\begin{abstract}
Background: This study aimed to evaluate the differences in alexithymia components between patients with obsessive-compulsive disorder $(\mathrm{OCD})$ and normal individuals.

Methods: In this ex-post facto study, 23 obsessive-compulsive outpatients' selected by available sampling method and 22 personnel of the same hospitals (Taft Comprehensive Psychiatric Hospital, Imam Ali, Shahid Rahnemoon, and Baghayipoor Clinics) were selected as a control group in 2015 in Yazd, Iran. Yale-Brown Obsessive-Compulsive and Toronto Alexithymia Scale-20 were used in this study.

Results: Data were analyzed by multivariate analysis of variance (MANOVA). The results showed that patients with OCD were significantly different from the control group in the total alexithymia score $(F=7.232, P=0.01)$. However, both groups had significant differences only in the subscale of difficulty in identifying feelings.

Conclusion: It can be concluded that both groups had significant differences in the total alexithymia score and one of its subscales.

Keywords: Obsessive-Compulsive Disorder (OCD); Alexithymia; Emotional Regulation
\end{abstract}

*Correspondence to Mina Bozorg,

Master of Clinical Psychology, Department of Clinical Psychology, Shiraz University (Eram Hill), Shiraz, Iran. Tel: +987136134662, Email: mina.b2369@yahoo. com

Published online 30 October 2021

Citation: Bozorg M, Rahimi C, Mohammadi N. Evaluating the components of alexithymia in patients with obsessive-compulsive disorder. Clin Neurosci J. 2021;8(4):175-179. doi:10.34172/icnj.2021.35.

\section{Introduction}

Obsessive-compulsive disorder (OCD) is described as one of the most common, disabling, and resistant disorders. This disorder is separated from the other anxiety disorders in the fifth edition of the Diagnostic and Statistical Manual of Mental Disorders (DSM-V). In this revision, a prominent feature of the disorder is obsessive and recurrent preoccupation. ${ }^{1}$ A widespread group of symptoms that include intrusive thoughts, rituals, preoccupations, and compulsions represents OCD.

OCD is a heterogeneous disorder with diverse symptoms. Extensive research that measures the heterogeneity of OCD focuses largely on certain maladaptive beliefs but many other factors such as emotion appraisal also predict the severity of OCD. ${ }^{2}$ A person with OCD diagnosis is unable to distinguish between imagined mental events and reality. This could heighten the distress associated with unpleasant thoughts and contribute to the sense that thoughts and reality are closely linked. ${ }^{3}$

People with OCD may have trouble clarifying their emotions, and naming their internal experiences (i.e., alexithymia), and these individuals may report experiencing "not just right" feelings (NJRFs). ${ }^{2}$ The difficulty in labeling and processing emotions may affect the representation of emotional states, and contribute to the clinical presentation of being fixed on particular topics and specific compulsive actions in OCD. ${ }^{3}$ During the past three decades, the construct of alexithymia has generated interest in identifying factors that might influence the cognitive processing of emotions and individual differences in levels of emotional awareness. ${ }^{4}$

The ability to identify and express emotions to process emotional experiences is necessary for having a long life. Alexithymia may interfere with the regulation of emotions. ${ }^{5}$ For example, cognitive reappraisal, and emotional regulation techniques contain mood state recognitions and situation interpretation to change a person's emotional reactions. ${ }^{6}$ One of the important factors for coping with negative emotions is identification and expression of emotions. Inability to understand the emotional experiences may make them more threatening.? Several studies have been conducted to support the fact that alexithymia is associated with OCD. One study showed that these patients have low awareness of their internal states. ${ }^{8}$ Loss of emotional expression leads to variety of social problems, psychological problems, and physical illness. $^{9}$

Besharat showed people with OCD had higher disabilities in all subscales of the alexithymia questionnaire. ${ }^{10}$ In recent literature, alexithymia is not 
mentioned as a psychiatric state. It is considered as a trait with a normal distribution in general population. ${ }^{11}$ Alexithymia is a major etiology for depression, OCD, substance abuse, and eating disorder. ${ }^{12}$ Bankier and colleagues found that extrovert thinking (a subscale of the Toronto alexithymia scale) is correlated with OCD. ${ }^{13}$ There was no significant change in the overall score which indicates that alexithymia is a fixed trait. ${ }^{14}$

Roh et al found a correlation between alexithymia and obsessive-compulsive symptoms. Differences between these two groups in alexithymia scale were significant. Low age and high level of anxiety were significantly associated with loss of emotion expression..$^{15}$ Deficits in emotion awareness are determined in patients with OCD. This inability causes social dysfunction. ${ }^{16}$

Awareness of one's feelings and ability to express them enable a person more to deal with life's challenges. This power improves mental health and on the other hand, loss of emotion expression leads to variety of social problems, psychological problems, and physical illness. We aimed to evaluate the differences in alexithymia components between patients with OCD and normal individuals.

\section{Materials and Methods \\ Participants}

This was an ex-post facto study done during spring 2015. Using convenience sampling method 23 adults aged 18-60 years with OCD were referred to the Taft Comprehensive Psychiatric Hospital, Imam Ali, Shahid Rahnemoon, and Baghayipoor Clinics in Yazd city, Iran. They were visited by a psychiatrist and received a diagnosis of OCD. After this step, they were assessed by Yale-Brown obsessivecompulsive scale (Y-BOCS) and if they had a score higher than nine, they were allocated to the OCD group. 22 healthy staff aged 18-60 were selected from these hospitals and clinics as the control group. The inclusion criteria for the OCD group were 18-60 years of age, junior education, and a score higher than nine in the Y-BOCS. Exclusion criteria for this group were the risk of other disorders in DSM-V and risk of neurological and other brain disorders. Inclusion criteria for the control group were similar to the patients group. Exclusion criteria for the healthy group were history of psychiatry disorders in themselves and history of OCD in their first-degree relatives.

\section{Procedure}

Patients who received the OCD diagnosis by a psychiatrist and a psychologist filled the consent form. After that, Y-BOCS and Toronto alexithymia scale were distributed randomly among the subjects. Patients could withdraw from the study at any time they desired. After collecting the patient group, a matched control group was selected. They also completed the Y-BOCS and Toronto Alexithymia scale. The two groups were compared afterwards.

\section{Instruments}

Y-BOCS: A semi-structured interview that has two parts: 1-Symptom Checklist (SC), 2- Severity Scale (SS). The 16 items of the self-report symptom checklist were answered on a 5-point Likert scale. The severity of symptoms was assessed in five dimensions of turmoil, frequency, interference, resistance, and control. Obsession severity, the severity of compulsion, and a total score that includes all items were marked.

The severity scale has 10 questions. First five questions show obsession severity and the next five questions determine the compulsion severity. Overall, 10 questions show the general OCD severity. The cut-off point of this scale is nine. Overall score below nine represents a low OCD, score between 8.99 and 14 indicates low intensity of OCD, 14 to 22.99 (moderate OCD), 23 to 27.99 (high intensity OCD), above 28 (very high intensity OCD). ${ }^{17}$ Internal validity for the two parts of the symptom checklist and severity scale are 0.97 and 0.95 respectively. Split-half reliability for the symptom checklist and severity scale is 0.93 and 0.89 . Test-retest reliability was 0.99 . There is a correlation between symptom checklist and SCL-90-ROCS $(P<0.001)$. There is a positive correlation between severity scale and SCID-I $(P<0.001)$. Exploratory factor analysis for SS and SC showed 3 and 2 factors respectively. ${ }^{18}$

Toronto Alexithymia Scale-20 (TAS): A 20 items selfreport scale, which has three dimensions: difficulty identifying feelings ( 7 items), difficulty expressing feelings (5 items), and externally-oriented thinking (8 items). The questions are based on a 5-point Likert scale from totally agree (1) to totally disagree (5). An overall score shows total alexithymia. Alpha coefficients for overall alexithymia, difficulty identifying feelings, difficulty expressing feelings, and externally oriented thinking are $0.85,0.82,0.75$, and 0.72 , respectively. Test-retest reliability after four weeks for this scale ranged from 0.8 to $0.87 .{ }^{19}$ Confirmatory factor analysis showed these three subscales in the Persian version of TAS.

\section{Statistical Process}

Descriptive methods including mean, standard deviation and inferential statistical method including multivariate analysis of variance (MANOVA) were used to compare the overall score of the variables in both healthy and patient groups. MANOVA was also used for comparing subscales of alexithymia in OCD and control group. All of these analyses were done with SPSS software, version 16.

\section{Results}

The average scores in the two subscales of alexithymia were higher in the OCD group, although the control group was better in externally oriented thinking subscale. The total score in patients with OCD were higher than the control group (Table 1). 
There was a significant difference in the alexithymia scale between the control and OCD groups $(\mathrm{F}=7.232, P=0.01)$.

The assumption of variance homogeneity between two groups was established in the two subscales of difficulty in identifying feelings and difficulty in expressing feelings but no variance homogeneity was observed in the externally oriented thinking subscale.

Wilk's lambda showed that multivariate analysis of variance between the two groups was significant with 95\% confidence. This indicated that alexithymia subscales differed significantly between the two groups (Table 2).

As shown in Table 3, there was a significant difference between the two groups in difficulty in identifying feelings. The sample size was less than 40 persons, so the univariate ANOVA was used.

\section{Discussion}

In this study, we compared differences in alexithymia subscales in the normal population and patients with OCD. Total alexithymia score in the OCD group had a higher score than the control group. This finding was consistent with the model of cybernetics for OCD. These

Table 1. Mean and Standard Deviation of Alexithymia Subscales in Control and OCD Groups

\begin{tabular}{lcccc}
\hline \multirow{2}{*}{ Alexithymia Subscale } & \multicolumn{2}{c}{ Control Group } & \multicolumn{2}{c}{ OCD Group } \\
\cline { 2 - 5 } & Mean & SD & Mean & SD \\
\hline Difficulty identifying feelings & 16.636 & 6.529 & 23.217 & 6.120 \\
Difficulty expressing feelings & 12.591 & 4.707 & 14.565 & 5.124 \\
Externally-oriented thinking & 21.182 & 3.984 & 21.130 & 5.941 \\
Total score & 50.41 & 10.26 & 58.91 & 10.92 \\
\hline
\end{tabular}

Table 2. Differences in Alexithymia Scale in Control and Patient Groups

\begin{tabular}{lcccc}
\hline \multirow{2}{*}{ Alexithymia Subscale } & \multicolumn{2}{c}{ Levene } & \multicolumn{2}{c}{ Wilks' Lambda } \\
\cline { 2 - 5 } & F & $\boldsymbol{P}$ & F & $\boldsymbol{P}$ \\
\hline Difficulty identifying feelings & 0.226 & 0.637 & & \\
Difficulty expressing feelings & 0.055 & 0.816 & 4.001 & 0.014 \\
Externally-oriented thinking & 4.923 & 0.032 & & \\
\hline
\end{tabular}

patients report a lack of correct emotions. ${ }^{20}$

Similar to another study, people with OCD had deficit in emotional awareness leading to social dysfunction ${ }^{4}$. Besharat indicated that the total score in alexithymia was high in the OCD group. ${ }^{10}$ Khosravani and colleagues determined that the patient group had a higher score in alexithymia. ${ }^{21}$ This finding was also consistent with another study. ${ }^{22}$

These findings suggest that people with OCD have difficulty identifying feelings. We found a significant relationship between difficulty expressing feelings and OCD disorder. These findings support the growing literature emphasizing that difficulty in identifying feelings is associated with obsessive-compulsive symptoms. ${ }^{23}$ Also, difficulty in this dimension and identifying feelings predispose people to emotional regulation deficits in stressful situations. ${ }^{24}$ It seems that OCD changes the cognitive processing ability of emotional information. Feeling, identifying, and understanding is necessary for emotion regulation skills. ${ }^{7}$ As we know reappraisal technique (one of the emotional regulation techniques) plays an important role in improving OCD. We revealed emotion recognition is impaired in patients with OCD, which was similar to many previous studies. Identifying and expressing feelings are important to tolerate negative ones. Inability in understanding the emotional experiences may make it threatening. ${ }^{8}$

We did not find the difficulty expressing feelings as a main predictor factor of OCD. To explain the results of neuropsychological approach we can say that cognitive stimulus that enters the right hemisphere, is transmitted to the left hemisphere by the corpus callosum. At first, the emotional dimension of the stimulus enters the limbic system and then goes through the left hemisphere through the anterior junction. Blocking of the corpus callosum may lead to alexithymia. At that time, the person feels the emotional experience but is not aware of it. ${ }^{25}$ Deficit in information processing is seen in OCD. Having the same neural foundation in alexithymia and OCD confirms the relationship between them. ${ }^{26}$ Patients with OCD have higher scores in all dimensions of alexithymia compared

Table 3: Univariate ANOVA in Alexithymia Subscales Between OCD and Control Groups

\begin{tabular}{|c|c|c|c|c|c|c|}
\hline \multirow{2}{*}{ Alexithymia Subscales } & & \multicolumn{5}{|c|}{ Index } \\
\hline & & SS & $d f$ & MS & $\mathbf{F}$ & $\boldsymbol{P}$ \\
\hline \multirow{2}{*}{ Difficulty identifying feelings } & Within group & 1719.004 & 43 & 39.997 & \multirow{2}{*}{12.182} & \multirow{2}{*}{0.001} \\
\hline & Between group & 486.996 & 1 & 486.996 & & \\
\hline \multirow{2}{*}{ Difficulty expressing feelings } & Within group & 1042.970 & 43 & 24.255 & \multirow{2}{*}{1.807} & \multirow{2}{*}{0.186} \\
\hline & Between group & 43.830 & 1 & 43.830 & & \\
\hline \multirow{2}{*}{ Externally-oriented thinking } & Within group & 1109.8881 & 43 & 25.811 & \multirow{2}{*}{0.001} & \multirow{2}{*}{0.973} \\
\hline & Between group & 0.030 & 1 & 0.030 & & \\
\hline
\end{tabular}

SS, Sums of Squares.

MS, Mean Squares. 
to the control group. We found this difference only in one dimension, difficulty identifying feelings. ${ }^{10}$ Difficulty in identifying and naming a person's feelings can drown the person in a sea of confusing experiences that resulted in concrete solutions to deal with OCD. ${ }^{24}$

The differences in the results indicate that OCD is a heterogeneous disorder that has different clinical features. Illness severity is important. Demographic information may differ in different studies and these factors may be effective in reaching a definite conclusion. The finding from the present study should also be considered within the context of several limitations. First, available sampling reduces the possibility of generalization. Selfreport instrument bias, lack of distinction between obsessions and compulsions are two main limitations of this research. It is suggested to perform a similar study to compare the obsessions and compulsions separately. Study of alexithymia based on severity and symptoms of OCD may be beneficial.

\section{Conclusion}

Patients with OCD have high scores in alexithymia and the difficulty identifying feelings subscale, these lead to difficulty identifying feelings. It causes lack of correct emotion reports which obligates them to repeat the work to achieve a true sense. It is assumed that OCD patients pay more attention to the physical senses because of the difficulty in identifying feelings. This increases the health concerns dimension of anxiety sensitivity.

Conflict of Interest Disclosures

The authors declare that they have no conflict of interests.

Ethical Statement

This study was derived from a thesis for the master's degree in clinical psychology and it was approved by Shiraz University (Code: 2720507).

\section{Acknowledgments}

We thank all hospitals and clinics that participated in this study for their support in data collection and management, Taft Comprehensive Psychiatry Hospital, Shahid Rahnemoon Clinic, Shahid baghayipoor Clinic and Imam Ali clinic. Special thanks to Dr. Bidaki and Dr. Nadi who helped in collecting data. Specially thanks to Bonnie Bozorg for assistance in editing the manuscript.

\section{References}

1. Brakoulias V, Starcevic V, Belloch A, Brown C, Ferrao YA, Fontenelle LF, et al. Comorbidity, age of onset and suicidality in obsessive-compulsive disorder (OCD): an international collaboration. Compr Psychiatry. 2017;76:7986. doi: 10.1016/j.comppsych.2017.04.002.

2. Smith AH, Wetterneck CT, Hart JM, Short MB, Björgvinsson T. Differences in obsessional beliefs and emotion appraisal in obsessive compulsive symptom presentation. J Obsessive Compuls Relat Disord. 2012;1(1):54-61. doi: 10.1016/j. jocrd.2011.11.003.

3. Robinson LJ, Freeston MH. Emotion and internal experience in obsessive compulsive disorder: reviewing the role of alexithymia, anxiety sensitivity and distress tolerance. Clin Psychol Rev. 2014;34(3):256-71. doi: 10.1016/j.cpr.2014.03.003.

4. Jansen M, Overgaauw S, De Bruijn ERA. Social cognition and obsessive-compulsive disorder: a review of subdomains of social functioning. Front Psychiatry. 2020;11:118. doi: 10.3389/fpsyt.2020.00118.

5. Smith R, Killgore WDS, Lane RD. The structure of emotional experience and its relation to trait emotional awareness: a theoretical review. Emotion. 2018;18(5):67092. doi: $10.1037 / \mathrm{emo} 0000376$.

6. Troy AS, Shallcross AJ, Brunner A, Friedman R, Jones MC. Cognitive reappraisal and acceptance: effects on emotion, physiology, and perceived cognitive costs. Emotion. 2018;18(1):58-74. doi: 10.1037/emo0000371.

7. Ong E, Thompson C. The importance of coping and emotion regulation in the occurrence of suicidal behavior. Psychol Rep. 2019;122(4):1192-210. doi: 10.1177/0033294118781855.

8. O'Kearney R, Nicholson C. Can a theory of mind disruption help explain OCD related metacognitive disturbances? Behav Change. 2008;25(2):55-70. doi: 10.1375/bech.25.2.55.

9. Van Kleef GA. The social effects of emotions are functionally equivalent across expressive modalities. Psychol Inq. 2017;28(2-3):211-6. doi: 10.1080/1047840X.2017.1338102.

10. Besharat MA. Psychometric characteristics of Persian version of the Toronto Alexithymia Scale-20 in clinical and non-clinical samples. Iran J Med Sci. 2008;33(1):1-6.

11. Xu P, Opmeer EM, van Tol MJ, Goerlich KS, Aleman A. Structure of the alexithymic brain: a parametric coordinatebased meta-analysis. Neurosci Biobehav Rev. 2018;87:50-5. doi: 10.1016/j.neubiorev.2018.01.004.

12. Hemming L, Haddock G, Shaw J, Pratt D. Alexithymia and its associations with depression, suicidality, and aggression: an overview of the literature. Front Psychiatry. 2019;10:203. doi: 10.3389/fpsyt.2019.00203.

13. Bankier B, Aigner M, Bach M. Alexithymia in DSMIV disorder: comparative evaluation of somatoform disorder, panic disorder, obsessive-compulsive disorder, and depression. Psychosomatics. 2001;42(3):235-40. doi: 10.1176/appi.psy.42.3.235.

14. Panasiti MS, Ponsi G, Violani C. Emotions, alexithymia, and emotion regulation in patients with psoriasis. Front Psychol. 2020;11:836. doi: 10.3389/fpsyg.2020.00836.

15. Roh D, Kim WJ, Kim CH. Alexithymia in obsessivecompulsive disorder: clinical correlates and symptom dimensions. J Nerv Ment Dis. 2011;199(9):690-5. doi: 10.1097/NMD.0b013e318229d209.

16. Apgáua LT, Jaeger A. Memory for emotional information and alexithymia A systematic review. Dement Neuropsychol. 2019;13(1):22-30. doi: 10.1590/1980$57642018 \mathrm{dn} 13-010003$.

17. Goodman WK, Price LH, Rasmussen SA, Mazure C, Fleischmann RL, Hill CL, et al. The Yale-Brown Obsessive Compulsive Scale. I. Development, use, and reliability. 
Arch Gen Psychiatry. 1989;46(11):1006-11. doi: 10.1001/ archpsyc.1989.01810110048007.

18. Rajezi Esfahani S, Motaghipour Y, Kamkari K, Zahiredin A, Janbozorgi M. Reliability and validity of the Persian version of the Yale-Brown Obsessive-Compulsive Scale (Y-BOCS). Iran J Psychiatry Clin Psychol. 2012;17(4):297303. [Persian].

19. Besharat MA. Reliability and factorial validity of a Farsi version of the 20-item Toronto Alexithymia Scale with a sample of Iranian students. Psychol Rep. 2007;101(1):20920. doi: 10.2466/pr0.101.1.209-220.

20. Thorsen AL, de Wit SJ, de Vries FE, Cath DC, Veltman DJ, van der Werf YD, et al. Emotion regulation in obsessivecompulsive disorder, unaffected siblings, and unrelated healthy control participants. Biol Psychiatry Cogn Neurosci Neuroimaging. 2019;4(4):352-60. doi: 10.1016/j. bpsc.2018.03.007.

21. Khosravani V, Samimi Ardestani M, Sharifi Bastan F, Kamali Z. The relationship between alexithymia and symptom dimensions in patients with obsessive-compulsive disorder. J Obsessive Compuls Relat Disord. 2017;14:127-33. doi: https://doi.org/10.1016/j.jocrd.2017.04.001.

22. Karami J, Momeni K, Zakiei A. The relationship alexitymia, positive affect and negative affect with the obsessive-compulsive disorder syndrome. Stud Med Sci. 2013;24(7):534-42. [Persian].

23. Pozza A, Giaquinta N, Dèttore D. The contribution of alexithymia to obsessive-compulsive disorder symptoms dimensions: an investigation in a large community sample in Italy. Psychiatry J. 2015;2015:707850. doi: 10.1155/2015/707850.

24. Cerutti R, Zuffianò A, Spensieri V. The role of difficulty in identifying and describing feelings in non-suicidal self-injury behavior (NSSI): associations with perceived attachment quality, stressful life events, and suicidal ideation. Front Psychol. 2018;9:318. doi: 10.3389/ fpsyg.2018.00318.

25. Goerlich-Dobre KS, Votinov M, Habel U, Pripfl J, Lamm C. Neuroanatomical profiles of alexithymia dimensions and subtypes. Hum Brain Mapp. 2015;36(10):3805-18. doi: 10.1002/hbm.22879.

26. Bagheri M, Nematollah Zadeh Mahani K, Pour Amrollahi $\mathrm{M}$. The mediating role of alexithymia in the relationship of coping strategies and personality traits with obsessivecompulsive disorder. Horizon Med Sci. 2020;27(1):62-81. doi: 10.32598/hms.27.1.3355.1. [Persian]. 